\title{
Intra-amniotic LPS causes acute neuroinflammation in preterm rhesus macaques
}

Augusto F. Schmidt ${ }^{1}$, Paranthaman S. Kannan' ${ }^{1}$, Claire A. Chougnet ${ }^{2}$, Steve C. Danzer ${ }^{3}$, Lisa A. Miller ${ }^{4}$, Alan H. Jobe ${ }^{1}$ and Suhas G. Kallapur ${ }^{1 *}$

\begin{abstract}
Background: Chorioamnionitis is associated with an increased risk of brain injury in preterm neonates. Inflammatory changes in brain could underlie this injury. Here, we evaluated whether neuroinflammation is induced by chorioamnionitis in a clinically relevant model.
\end{abstract}

Methods: Rhesus macaque fetuses were exposed to either intra-amniotic (IA) saline, or IA lipopolysaccharide (LPS) ( $1 \mathrm{mg}) 16$ or $48 \mathrm{~h}$ prior to delivery at 130 days ( $85 \%$ of gestation) ( $n=4-5$ animals/group). We measured cytokines in the cerebrospinal fluid (CSF), froze samples from the left brain for molecular analysis, and immersion fixed the right brain hemisphere for immunohistology. We analyzed the messenger RNA (mRNA) levels of the pro-inflammatory cytokines IL-1 $\beta, C C L 2, T N F-a, I L-6, I L-8, I L-10$, and COX-2 in the periventricular white matter (PVWM), cortex, thalamus, hippocampus, and cerebellum by RT-qPCR. Brain injury was assessed by immunohistology for myelin basic protein (MBP), IBA1 (microglial marker), GFAP (astrocyte marker), OLIG2 (oligodendrocyte marker), NeuN (neuronal marker), CD3 ( $T$ cells), and CD14 (monocytes). Microglial proliferation was assessed by co-immunostaining for IBA1 and Ki67. Data were analyzed by ANOVA with Tukey's post-test.

Results: IA LPS increased mRNA expression of pro-inflammatory cytokines in the PWWM, thalamus, and cerebellum, increased IL-6 concentration in the CSF, and increased apoptosis in the periventricular area after $16 \mathrm{~h}$. Microglial proliferation in the white matter was increased $48 \mathrm{~h}$ after IA LPS.

Conclusions: LPS-induced chorioamnionitis caused neuroinflammation, microglial proliferation, and periventricular apoptosis in a clinically relevant model of chorioamnionitis in fetal rhesus macaques. These findings identify specific responses in the fetal brain and support the hypothesis that neuroinflammatory changes may mediate the adverse neurodevelopmental outcomes associated with chorioamnionitis.

Keywords: Chorioamnionitis, Prematurity, Brain injury, Periventricular leukomalacia, Cytokines, Microglia, Apoptosis Abbreviations: CCL2, Chemokine ligand 2; CD, Cluster differentiation; CNPRC, California National Primate Research Center; CSF, Cerebrospinal fluid; COX-2, Cyclooxygenase-2; CP, Cerebral palsy; ELISA, Enzyme-linked immunosorbent assay; GFAP, Glial fibrillary acidic protein; Iba1, Ionized calcium-binding adaptor molecule 1; IA, Intra-amniotic; IL, Interleukin; Ki-67, Marker of proliferation; MBP, Myelin basic protein; MRI, Magnetic resonance imaging; NeuN, Neuronal nuclei; Olig2, Oligodendrocyte transcription factor 2; LPS, Lipopolysaccharide; PBS, Phosphate buffer saline; PTGES2, Prostaglandin E synthase 2; PVL, Periventricular leukomalacia; PWWM, Periventricular white matter; TNF, Tumor necrosis factor; WBC, White blood cell count

\footnotetext{
* Correspondence: suhas.kallapur@cchmc.org

'Division of Neonatology and Pulmonary Biology, Cincinnati Children's

Hospital Medical Center, 3333 Burnet Ave, Cincinnati, OH 45229, USA

Full list of author information is available at the end of the article
} 


\section{Background}

Chorioamnionitis is frequently associated with preterm birth [1], and fetal exposure to inflammation is an independent risk factor for brain injury, including intraventricular hemorrhage (IVH), periventricular leukomalacia (PVL), cerebral palsy $(\mathrm{CP})$, and cognitive impairment $[2,3]$. In a multicenter prospective study of 3094 infants born before 33 weeks' gestational age, clinical chorioamnionitis increased the risk of severe intraventricular hemorrhage, with an odds ratio of 1.62 [4]. Histological chorioamnionitis increased the risk of intraventricular hemorrhage, periventricular leukomalacia, and cerebral palsy on neurodevelopmental testing between 30 and 42 months corrected age (odds ratio 2.45) in a recent retrospective study with prospective follow-up including infants born with less than 29 weeks gestational age [5].

Selection of the animal model is a critical variable for research examining chorioamnionitis-induced brain injury. The most commonly used model for inflammation induced brain injury combines hypoxia/ischemia and intraperitoneal lipopolysaccharide (LPS) in term newborn rodents [6]. Neuroinflammation, characterized by microglial activation and damage to the white matter, is consistently observed in this model [7]. Additionally, preterm infants with exposure to histological chorioamnionitis have only modest increases in inflammatory cytokines in the serum and do not have manifestations of systemic inflammatory response [8].

Systemic inflammation can cause an inflammatory response in the fetal brain with microglial activation, upregulation of cytokines, and neuronal apoptosis $[9,10]$. In neonatal rats, intra-cerebral injection of IL- $1 \beta$ induces apoptosis and astrogliosis and decreases myelin basic protein (MBP) staining [11]. The IL-1 $\beta$ injection results in long-term neuro-inflammatory responses and motor behavioral deficits, which are attenuated by an IL-1 receptor antagonist [12]. Another cytokine that has been implicated in the pathogenesis of brain injury in preterm infants is IL-6. Increased IL-6 concentrations in the cord blood of preterm newborns are associated with increased risk of white matter damage, germinal matrix hemorrhage, and cystic lesions $[13,14]$. Brain injury secondary to diverse insults such as trauma, excitatory injury, and infection is also associated with increased expression of COX-2, but there are no data regarding its role in brain injury associated with chorioamnionitis [15].

Inflammation in human chorioamnionitis is largely localized to the uterus, without overt fetal hypoxia or strong maternal systemic inflammatory responses. In fact, measurements of systemic inflammatory markers such as procalcitonin, CRP and IL-6 in the maternal serum do not identify intrauterine inflammation $[16,17]$.
In contrast to intraperitoneal or systemic LPS injections, intra-amniotic (IA) injections of pro-inflammatory mediators such as IL-1 $\beta$ or LPS induce a predominantly chorioamniotic inflammation with subtle fetal systemic inflammation, similar to the pathology in humans [18]. In preterm fetal sheep, IL-1 $\beta$ was upregulated in the hippocampus, cortex, and cerebellum 2 days after exposure to IA LPS [19], followed by microglial and astrocyte recruitment, apoptosis, and decreased myelination of the subcortical white matter and hippocampus after 7 days [20]. Nonetheless, although studies in rodents and sheep provide some insight into the human pathology, there are striking differences in neurological development and vulnerability to brain injury between these species and humans.

In humans, generation of oligodendrocytes peaks between 23 and 32 weeks of gestation, while in rodents, peak oligodendrogenesis is delayed until 1 to 3 days after birth. Similarly, peak brain growth and gliogenesis occur between 36 and 40 weeks' gestation in humans, whereas in rodents, the peak occurs between postnatal days 7 to 10 [21]. In contrast to rodents, where development is delayed relative to humans, sheep develop at a faster rate than humans. In sheep, $50 \%$ of the adult brain weight is achieved at $80 \%$ of gestation, while humans do not reach this milestone until early infancy. Similarly, the internal capsule myelination starts at $70 \%$ of gestation in sheep and not until 40 days postnatally in humans [22]. In contrast, rhesus macaques reach $50 \%$ of adult brain weight at term and peak internal capsule myelination starts at $90 \%$ gestation, closer to human development. Thus, nonhuman primate models, such as rhesus macaques, are especially attractive due to similar ontogeny of the nervous systems [22].

We tested the hypothesis that IA administration of LPS will induce inflammation in the central nervous system in the rhesus macaque.

\section{Methods \\ Animals}

The Institutional Animal Care and Use Committee at the University of California Davis approved all animal procedures, which were performed at the California National Primate Research Center (CNPRC), University of California, Davis. Time-mated pregnant rhesus macaques were sedated with ketamine at approximately day 130 of gestation (term is 165 days) for ultrasound exam and IA injection of either LPS (1 mg, E. coli O55:B5, Sigma Aldrich, Saint Louis, MO) diluted in $1 \mathrm{~mL}$ of sterile saline solution or $1 \mathrm{~mL}$ of sterile saline as the control injection. After 16 or $48 \mathrm{~h}$, fetuses were surgically delivered for fetal tissue collection. We collected cerebrospinal fluid (CSF) by occipital puncture. The left brain was dissected and the cortex, periventricular white 
matter (PVWM), thalamus, hippocampus, and cerebellum were snap frozen for molecular analysis. The right brain was fixed in $10 \%$ formalin. We used four to five animals for each group.

\section{mRNA quantitation}

Total RNA was isolated from frozen brains after homogenization with TRIzol (Invitrogen, Carlsbad, CA) and column purified with RNeasy Universal MiniKit (Qiagen, Valencia, CA) according to the manufacturer's instructions. Reverse transcription was performed using Verso complementary DNA (cDNA) kit (Thermo Scientific, Waltham, MA) to produce single-strand cDNA. The genes for IL- $1 \beta$, CCL2, TNF- $\alpha$, IL-6, IL-8, IL-10, cyclooxygenase-2 (COX-2), and prostaglandin E synthase 2 (PTGES2) were amplified by RT-PCR using the cDNA template and rhesus macaque-specific primers along with Taqman probes (Applied Biosystems, Foster City, CA). The messenger RNA (mRNA) expression for each gene was normalized to the mRNA for the ribosomal protein $18 \mathrm{~s}$ as internal standard. Data are expressed as fold increase over the mean control value.

\section{Cytokine measurements}

We measured cytokine concentrations in the CSF by Luminex using nonhuman primate multiplex kits (Millipore, Billerica, MA) according to the manufacturer's protocol. Concentrations were calculated from standard curves using recombinant proteins and expressed in picograms per milliliter.

\section{Immunohistochemistry}

Sections from formalin fixed tissues in paraffin blocks were deparaffinized and rehydrated before microwaveassisted antigen retrieval in citric acid buffer at $\mathrm{pH}$ 6.0. Endogenous peroxidase activity was reduced with $\mathrm{CH}_{3} \mathrm{OH} / \mathrm{H}_{2} \mathrm{O}_{2}$ treatment, and the tissue was blocked with $2 \%$ donkey serum in phosphate buffer saline (PBS). Sections were incubated overnight at $4{ }^{\circ} \mathrm{C}$ with the primary antibody diluted in $2 \%$ serum in PBS. We used the following primary antibodies: goat polyclonal AntiIba1 (Abcam, Cambridge, MA, cat. \#5076, dilution 1:200), rabbit monoclonal Anti-glial fibrillary acidic protein (GFAP) (Abcam, Cambridge, MA, cat. \#48050, dilution 1:500), rabbitt monoclonal Anti-Olig2 (Abcam, Cambridge, MA, cat. \#109186, dilution 1:500), mouse monoclonal Anti-MBP (Abcam, Cambridge, MA, cat. \#62631, dilution 1:500), rabbit monoclonal Anti-Tau (Abcam, Cambridge, MA, cat. \#32057, dilution 1:500), mouse monoclonal anti-NeuN (Abcam, Cambridge, MA, cat. \#ab104224, dilution 1:200), rabbit polyclonal antiCD3 (DakoCytomation, Carpinteria, CA, cat. \#A0452, dilution 1:100), and mouse monoclonal anti-CD14 (BD Biosciences, San Diego, CA, cat. \#557742, dilution
1:100). Sections were then washed and incubated with the appropriate species-specific secondary antibody diluted 1:200 in $2 \%$ serum for $2 \mathrm{~h}$ at room temperature. After further washing, antigen/antibody complexes were visualized using a Vectastain $\mathrm{ABC}$ peroxidase kit (Vector Laboratories Inc., Burlingame, CA). Antigen detection was enhanced with nickel-diaminobenzidine, followed by incubation with Tris-cobalt. Slides were counterstained with Nuclear Fast Red for photomicroscopy.

\section{Double-labeling immunofluorescence}

To identify proliferating microglia, we performed doublelabeling immunofluorescence for Iba-1 and Ki-67. Antigen retrieval and blocking were conducted as described above, followed by overnight incubation with goat polyclonal Anti-Iba1 (Abcam, Cambridge, MA, cat. \#5076, dilution 1:100) and rat monoclonal anti-Ki67 (LifeSpan Biosciences, Seattle, WA, cat. \#LS-C175347, dilution 1:50). The following day, sections were washed and incubated with antigoat Alexa Fluor 594 (Life technologies, Carlsbad, CA, cat. \#A-11058, dilution 1:200) and anti-rat Alexa Fluor 488 (Life technologies, Carlsbad, CA, cat. \#A-21470, dilution 1:200) for $2 \mathrm{~h}$ at room temperature, followed by washing and incubation with DAPI (Life technologies, Carlsbad, CA, cat. \#D1306, dilution 1:2000) for $15 \mathrm{~min}$ at room temperature. Sections were washed and mounted with ProLong Gold (Life technologies, Carlsbad, CA, cat. \#P36930). Stained slides were imaged on confocal microscopy for colocalization at $\times 40 x$ magnification with $1024 \times 1024$ pixel resolution on a Nikon Eclipse A1RSi inverted microscope (Nikon Instruments Inc, Melville, NY).

\section{Cell counting}

Cells immunostained for CD3 (T cells), CD14 (monocytes), and caspase-3 (apoptotic cells) were counted in six randomly selected fields per animal on photomicrographs $(\times 40)$ from the periventricular area and counted by a masked investigator. A similar strategy was used to assess the number (Iba1+) and proportion of proliferating microglia (Ki67+/Iba1+) in the white matter. A masked investigator counted the number of cells positive for Iba1, and the number of cells positive for both Iba1 and Ki67 in each determined both the number of Iba1+ cells and the percentage of Iba $1+/ \mathrm{Ki} 67+$ cells.

\section{Statistical analysis}

Results of the mRNA quantitation and cell counting were analyzed by ANOVA followed by post-testing comparison of each experimental group against the control group with Tukey correction. Statistical analysis was performed on GraphPad Prism version 6.0 for Mac OS X (GraphPad Software, San Diego, CA). Data are presented as means \pm standard deviation. Values of $p<0.05$ were considered significant. 
Table 1 Animal data

\begin{tabular}{llcclccc}
\hline & $n$ & Gestational age (days) & Birth weight (grams) & Sex (M/F) & WBC $\left(10^{9} / L\right)^{*}$ & Neutrophils (\%) & Bands (\%) \\
\hline Control & 5 & $132 \pm 2$ & $343 \pm 9$ & $3 / 2$ & $2.0 \pm 0.9$ & $9 \pm 3$ & 0 \\
LPS 16 h & 5 & $131 \pm 1$ & $333 \pm 43$ & $2 / 3$ & $2.0 \pm 0.4$ & $14 \pm 15$ & $1.5 \pm 1^{*}$ \\
LPS 48 h & 4 & $130 \pm 2$ & $307 \pm 17$ & $0 / 4$ & $3.3 \pm 1.7$ & $25 \pm 10$ & $1 \pm 1$ \\
\hline
\end{tabular}

WBC white blood cell count

${ }^{*} p<0.05$

\section{Results}

\section{Animals}

Gestational ages at birth and birth weights were similar among the animals (Table 1). One animal in the LPS $48 \mathrm{~h}$ group was a fetal death and was excluded from the study. There was a non-significant trend towards increased cord white blood cell and neutrophil counts after IA LPS. The percentage of band form of neutrophils significantly increased in the LPS $16 \mathrm{~h}$ group compared to control $(p<0.05)$.

\section{IA LPS increases cytokine expression in the brain}

In the PVWM, IL-1 $\beta$ mRNA increased by twofold 16 and $48 \mathrm{~h}$ after IA LPS $(p<0.05)$ (Fig. 1). In the cerebellum, IL-1 $\beta$ and COX-2 mRNA expression increased $16 \mathrm{~h}$ after IA LPS $(p<0.05)$. In the thalamus, expression of CCL2 and COX-2 mRNA increased in the LPS $16 \mathrm{~h}$ group $(p<0.05)$. There were no changes in expression of IL-6, IL-10, or PTGES2 in the
PVWM, cerebellum, or thalamus (Additional file 1). IL-8 mRNA expression increased in the hippocampus $16 \mathrm{~h}$ after IA LPS, but no other cytokines were increased. Cytokine mRNA levels were unchanged in the cortex (Additional file 2).

\section{IA LPS increases concentration of IL- 6 concentration in} the plasma and CSF

IL-6 significantly increased in both the plasma and CSF after IA LPS exposure. In plasma, a 300-fold increase over controls was observed, while a more modest 4-fold increase was evident in the CSF (Fig. 2). The concentration of CCL2 was also increased in the fetal plasma at $16 \mathrm{~h}$ but not in the CSF $16 \mathrm{~h}$ after IA LPS exposure (Fig. 2). There were no differences in the levels of other cytokines including IL-1 $\beta$, IL-8, IL10 , and TNF- $\alpha$ in the CSF or in the fetal plasma (Additional file 3).

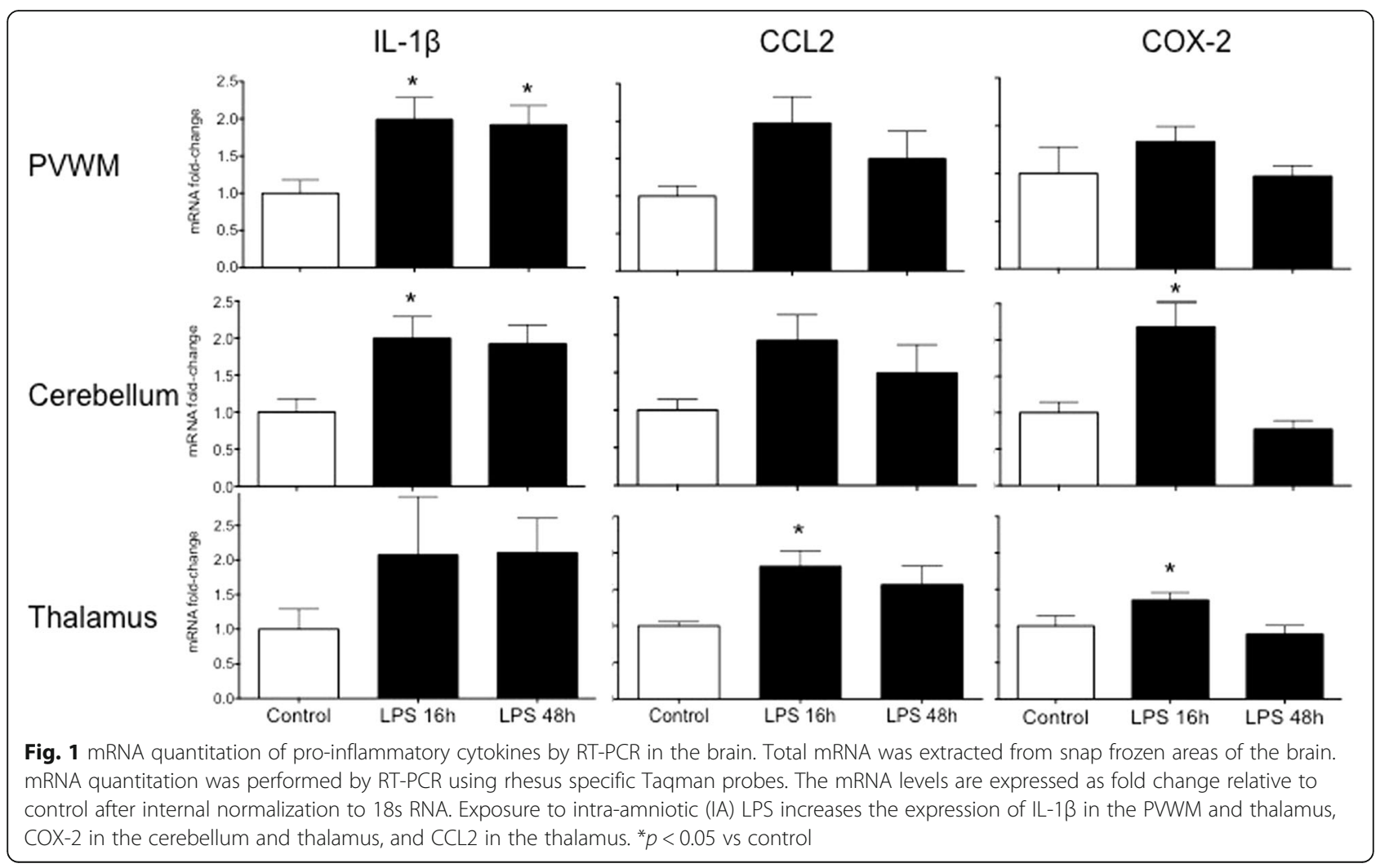



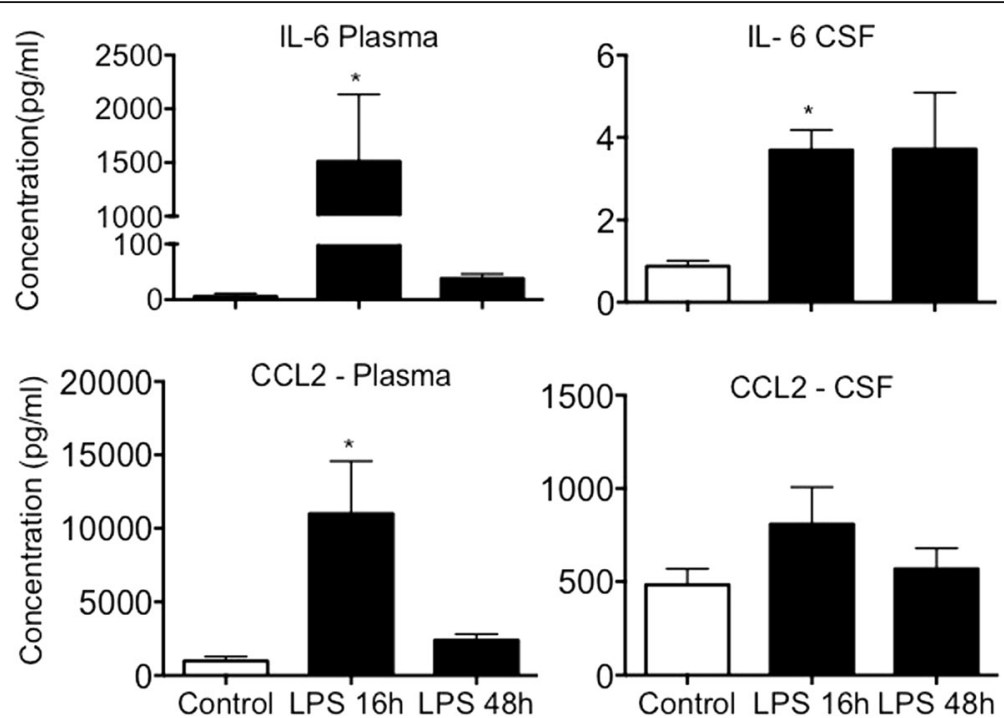

Fig. 2 IL-6 concentration in the fetal plasma and CSF measured by ELISA. Exposure to IA LPS significantly increased the concentration of IL-6 in the plasma and CSF. ${ }^{*} p<0.05$ vs control

\section{IA LPS induces apoptosis in the white matter}

We performed immunohistochemistry for activated caspase 3 in order to identify cells undergoing apoptosis. Caspase 3 positive cells were significantly increased in the periventricular white matter $16 \mathrm{~h}$ after IA LPS $(p<0.05)$, which lowered at $48 \mathrm{~h}$ after IA LPS (Fig. 3). There were no differences in the intensity or pattern of immunostaining for Iba-1, GFAP, MBP, Olig2, Tau, and NeuN (Fig. 4). We did not observe infiltrating leukocytes (CD3+) or infiltrating monocytes (CD14+) in the brain after IA LPS (Fig. 5).

\section{IA LPS induces proliferation of microglia}

In order to identify and count the proportion of proliferating microglia in the white matter, we performed double-labeling immunofluorescence for Iba-1 and Ki67. We observed an increased percentage of proliferating microglia $48 \mathrm{~h}$ after exposure to IA LPS ( $p<0.05$, Fig. 6).

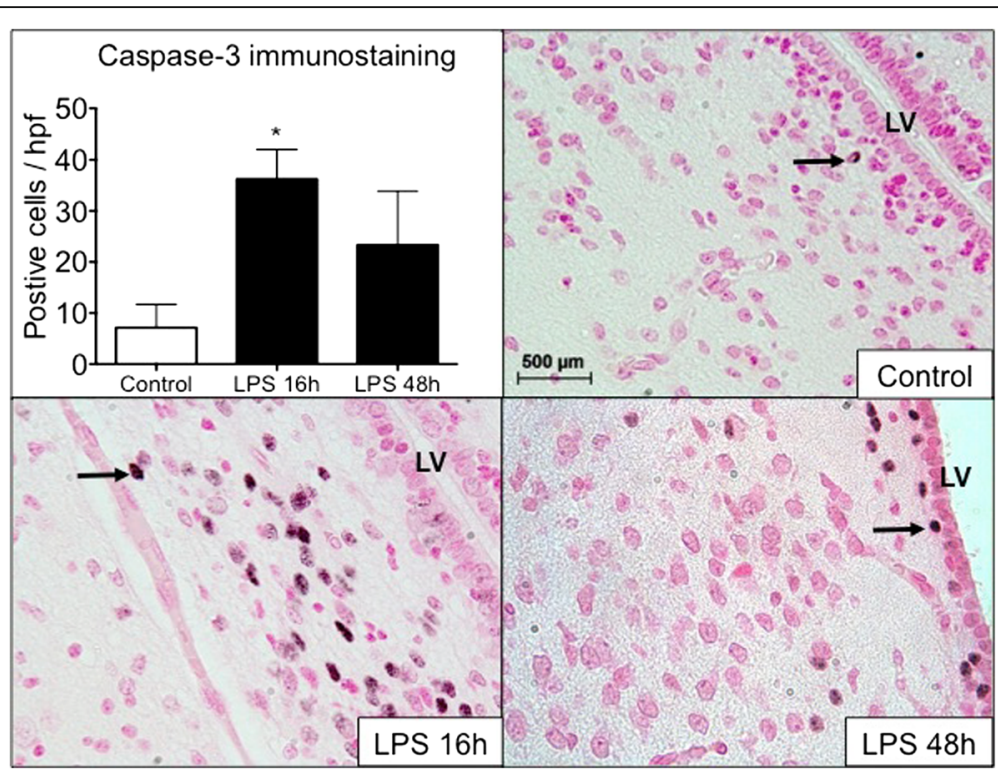

Fig. 3 Immunohistochemical staining for activated caspase-3 in the ventricular zone. Representative sections of paraffin-embedded brain tissue from rhesus macaques fetus. Immunohistochemical staining for activated caspase-3 in the ventricular zone (arrows). Exposure to IA LPS increases the number of activated caspase-3 positive cells in the ventricular zone $16 \mathrm{~h}$ after IA LPS. Representative micrographs show the immunostaining pattern for activated caspase-3 for control, LPS $16 \mathrm{~h}$, and LPS $48 \mathrm{~h}(\times 40)$. LV lateral ventricle. ${ }^{*} p<0.05$ vs control 


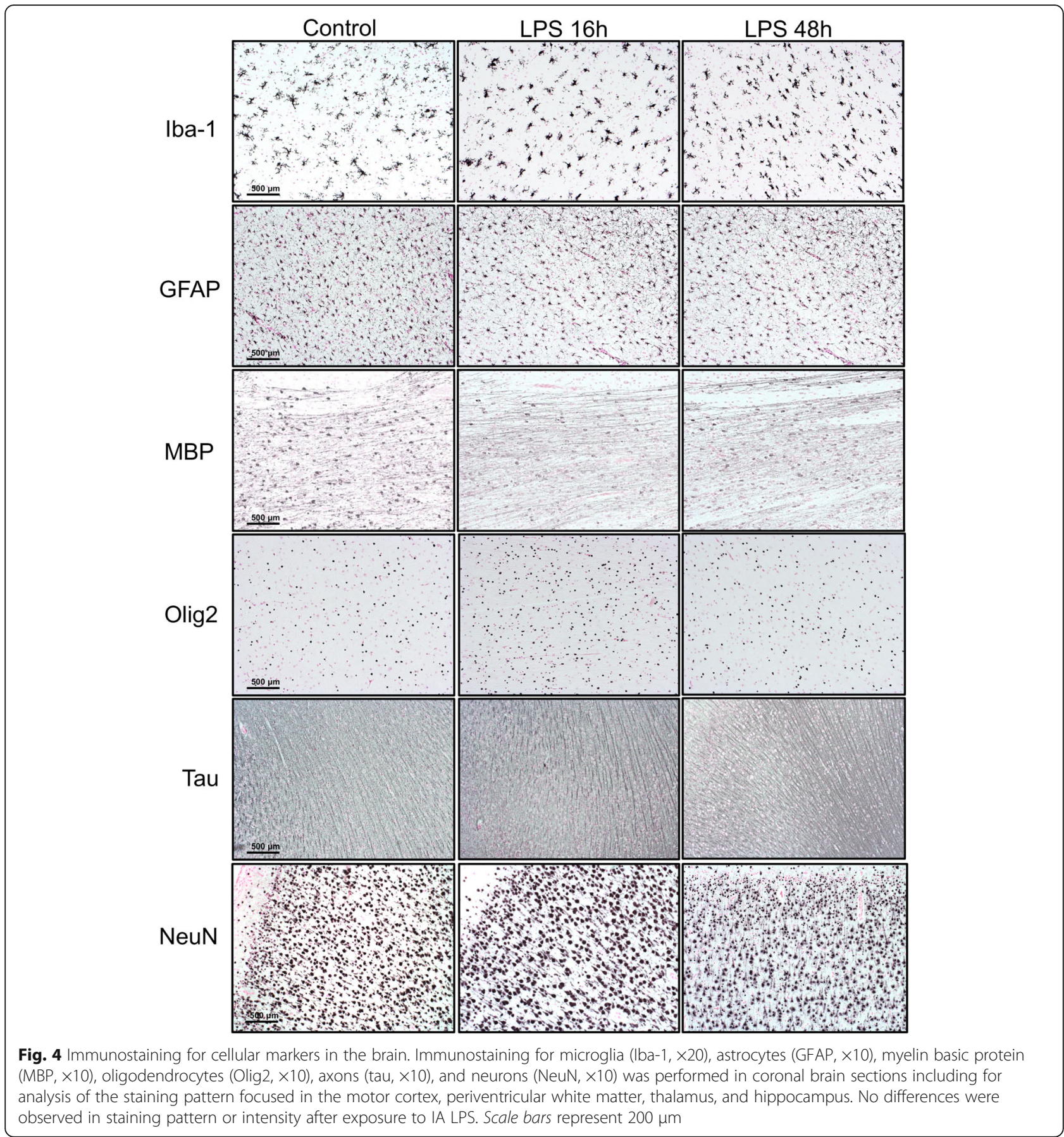

\section{Discussion}

Prematurity is the most common cause of infant mortality in the USA [23] and is frequently associated with chorioamnionitis, with at least $40 \%$ of preterm births having prenatal inflammation [24]. Moreover, chorioamnionitis is an independent risk factor for brain injury in the neonatal period including IVH and PVL and late neurodevelopmental impairment such as $\mathrm{CP}$ and cognitive deficits [25-28]. Even in the absence of prematurity or signs of early neonatal brain injury such as IVH and
PVL, chorioamnionitis is still associated with increased risk of CP [29]. We report the novel finding of neuroinflammatory responses in a nonhuman primate model of chorioamnionitis induced by IA LPS. Our data suggest that neuroinflammation is a likely consequence of the fetal inflammatory response syndrome.

Even though the association between systemic inflammation and brain injury is well known, the specific mechanisms through which circulating cytokines lead to microglial activation and increased expression of 


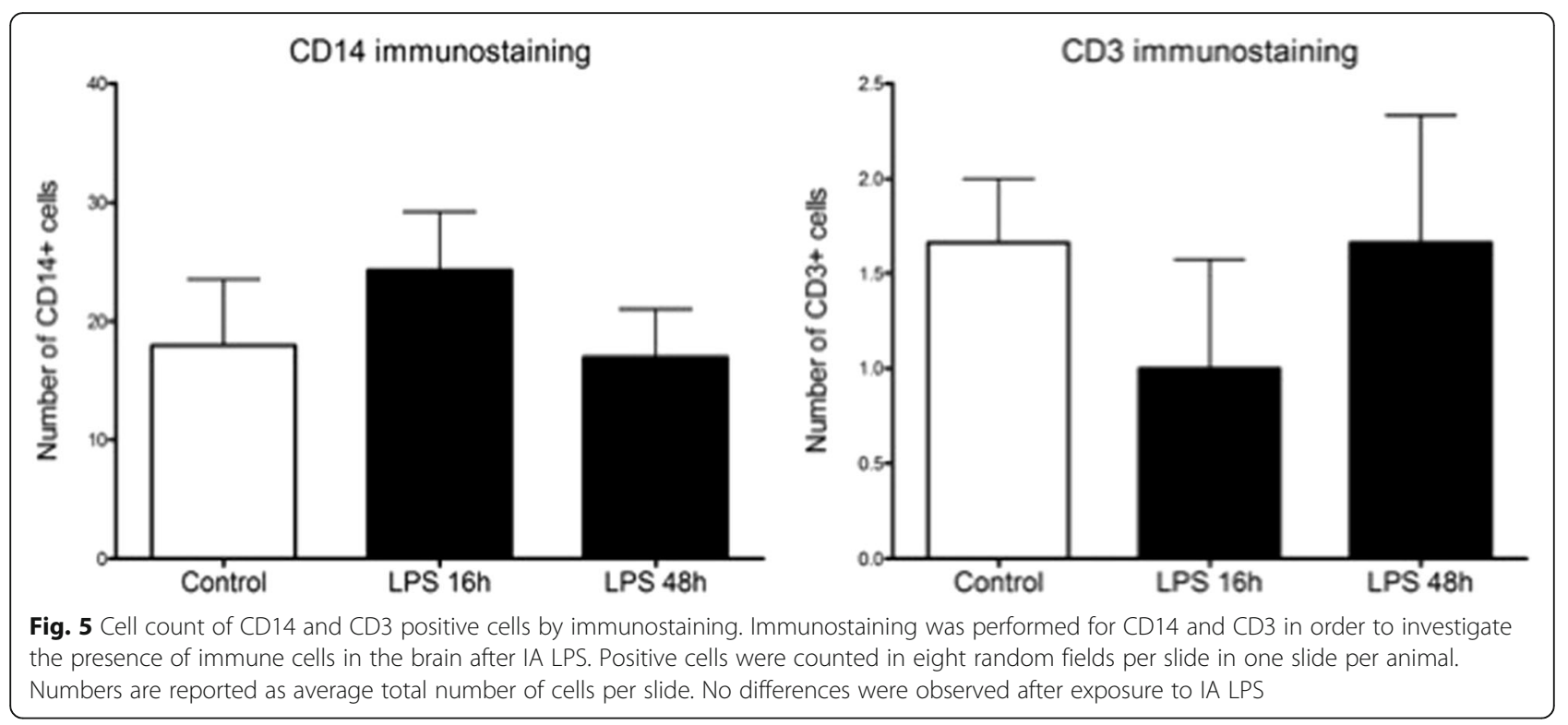

cytokines in the central nervous system remain elusive [30]. We measured increased levels of IL-6 in the plasma and CSF of animals exposed to IA LPS. Increased IL-6 levels in cord blood and in the amniotic fluid have been correlated with intrauterine infection/inflammation and fetal inflammatory response syndrome [14, 31, 32]. Our data showing a significant gradient between plasma and CSF IL-6 is consistent with IL-6 crossing the bloodbrain barrier through a saturable transport system [33]. Even though the plasma concentration of CCL2 was increased in LPS exposed animals, there was no significant increase in the CSF concentration of this cytokine.

The increased circulating IL-6 may cross the bloodbrain barrier to the CSF. IL- 6 in the central nervous system has the potential to activate the resident microglia and trigger a local inflammatory response with upregulation of pro-inflammatory cytokines. In one study of 146 preterm infants, the presence of higher CSF concentrations of IL-6, IL-10, and TNF- $\alpha$ was associated with white matter injury identified by magnetic resonance imaging (MRI) at term equivalent age [13]. However, there was no relationship between CSF cytokines and plasma cytokine concentrations. Elevated plasma CCL2 can also mediate the neuro-inflammatory response. CCL2, and its receptor have been implicated in the pathogenesis of brain injury due to trauma and autoimmune processes $[34,35]$. Interestingly, the CCL2 levels in the CSF may be reduced in autoimmune conditions due to consumption by CCR2+ leukocytes [36]. After IA LPS, CCL2 concentration did not increase in the fetal CSF and there was no infiltration of leukocytes.
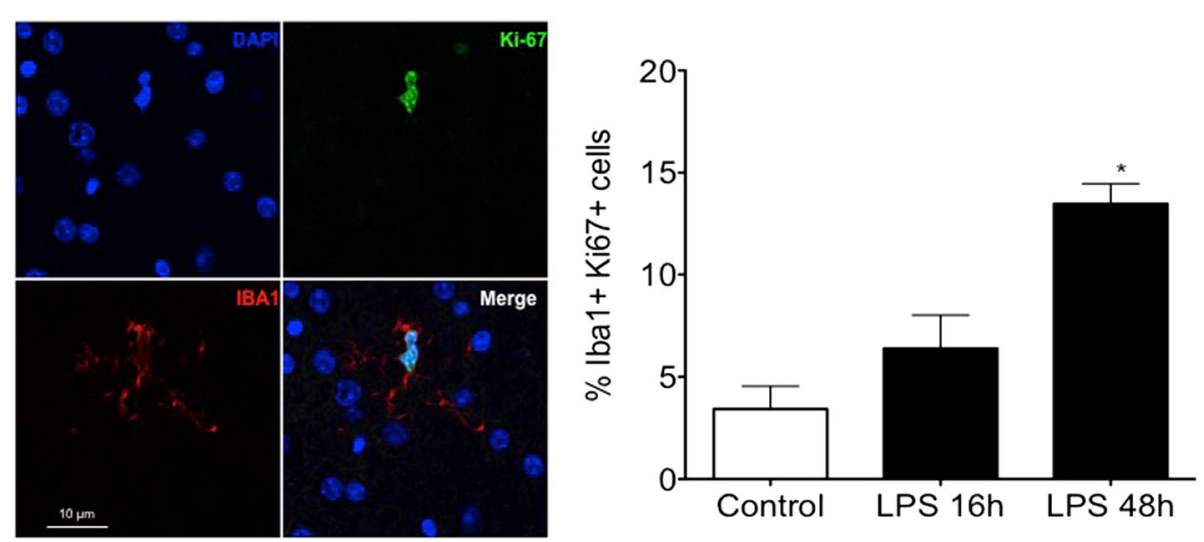

Fig. 6 Double-labeling immunofluorescence for Iba-1 and Ki-67 and percentage of microglia labeling for Ki-67. Representative sections of paraffin-embedded brain tissue from rhesus macaques fetus. Confocal microscopy showing a microglia labeled for nuclei (blue), Iba-1 (red), and Ki-67 (green) (×60). Relative number of proliferating microglia: percentage of Iba-1 positive cells that were co-stained for Ki-67. ${ }^{*} p<0.05$ vs control 
The elevation of IL-1 $\beta$ in the PVWM at 16 and $48 \mathrm{~h}$ after IA LPS may be especially important in the pathogenesis of white matter injury associated with chorioamnionitis. In preterm infants, injury to pre-myelination oligodendrocytes is central to the pathogenesis of white matter injury [11]. The expression of IL- $1 \beta$ and COX-2 mRNA was increased in the cerebellum $16 \mathrm{~h}$ after IA LPS. Although the cerebellum is not classically considered a site of brain injury in premature infants, more recent findings indicate that cerebellar underdevelopment may be common in preterm infants [37]. A strong relationship between decreased cerebellar volumes with presence of PVL has been reported [38], suggesting a common insult, such as inflammation, which is known to cause injury to the developing cerebellum [39, 40]. Another site with upregulation of pro-inflammatory cytokines after IA LPS was the thalamus, where we observed a twofold increase in CCL2 and COX-2 mRNA and a trend towards increased IL-1 $\beta$ mRNA. Neuropathological studies of premature infants show greater neuronal loss in the thalamus compared to the cortex and a higher incidence of thalamic injuries in the presence of PVL $[41,42]$. Due to its projections to the cerebral cortex and the role of the thalamus in cognitive and social function, thalamic damage in PVL could contribute to the encephalopathy of prematurity.

COX-2 has been investigated in neonatal brain following hypoxic ischemic insults [43], but there are no data regarding a potential role of this enzyme and prostaglandins in the pathogenesis of encephalopathy of prematurity. The inducible form of COX-2 is polymorphic and the allele associated with decreased gene expression and decreased synthesis of prostaglandin is independently associated with worse long-term cognitive outcomes in preterm infants [44]. The use of the nonspecific cyclooxygenase inhibitor indomethacin in preterm newborns was associated with decreased incidence of PVL, even though indomethacin does not change long-term neurodevelopmental outcomes [45]. We found increased expression of COX-2 mRNA in the thalamus and cerebellum $16 \mathrm{~h}$ after exposure to IA LPS, suggesting that COX-2 may also be implicated in the brain injury induced by prenatal inflammation.

We also detected microglial proliferation after fetal exposure to IA LPS that starts at $16 \mathrm{~h}$ and is significant $48 \mathrm{~h}$ after the initial insult. Microglia are important during brain development with a variety of roles including but not limited to neuronal proliferation and differentiation, synaptic remodeling, and myelination [46]. Microglia also have a central role in the inflammatory response in the central nervous system, and PVL is associated with marked microgliosis [47]. Once activated, microglia release cytokines that can injure preoligodendrocyte populations [48]. It is interesting that the largest increase in microglial proliferation was after $48 \mathrm{~h}$ of IA LPS exposure, while cytokine mRNAs were increased at $16 \mathrm{~h}$. The continued microglial proliferation could result in long-term consequences to the development of brain as has been observed in rodents models, where a single dose of intra-cerebral LPS leads to motor behavioral changes through adulthood [11].

IA LPS increased apoptosis in the periventricular white matter, as demonstrated by immunostaining for activated caspase-3. This area is a frequent site of injury in preterm infants [3]. Our findings are consistent with other models of preterm and neonatal brain injury. In rodents, intra-cerebral LPS induces apoptosis in the white matter [49]. In the sheep model, IA LPS increases the number of apoptotic cells in the PVWM and cortical area, with associated microglial activation $[20,50]$. In this model, longer exposure to IA LPS resulted in increased injury and abnormalities on EEG with increased delta wave frequency after 14 days [50]. The association of IA LPS, early expression of inflammatory markers in the brain, and late functional changes supports the idea that exposure to prenatal inflammation causes specific inflammatory responses in the brain and may result in the adverse neurodevelopmental outcomes observed in preterm newborns exposed to chorioamnionitis.

\section{Conclusions}

IA injection of LPS in a nonhuman primate results in modest acute inflammatory responses in the central nervous system evidenced by increased cytokine in the CSF and cytokine mRNA levels and microglial proliferation in the brain. The inflammatory response is associated with apoptosis in the periventricular white matter. This novel model of fetal brain injury induced by chorioamnionitic inflammation should be useful for understanding the pathogenesis of white matter injury associated with prenatal inflammation. Our results suggest that the brain injury in fetuses exposed to chorioamnionitis could be mediated by circulating IL- 6 and/or CCL2.

\section{Additional files}

Additional file 1: mRNA quantitation of $I L-6, I L-10$, and PTGES2 in the PWWM, cerebellum, and thalamus. (DOCX $69 \mathrm{~kb}$ )

Additional file 2: mRNA quantitation of pro-inflammatory cytokines in the hippocampus and cortex. (DOCX $14 \mathrm{~kb}$ )

Additional file 3: Cytokine concentrations in the fetal plasma and fetal cerebrospinal fluid (CSF) measured by ELISA. (DOCX 54 kb)

\section{Acknowledgements}

Not applicable.

\section{Funding}

The funding sources for this study are Cincinnati Children's Hospital Medical Center-Arnold Strauss Fellows' Award (A.F.S), March of Dimes Innovation catalyst award (S.G.K), Burroughs-Wellcome Fund, and Preterm Initiative (C.A.C.).

The funding agencies had no role in the design, collection, analysis, or interpretation of the data and in the writing the manuscript. 


\section{Availability of data and materials}

The datasets during and/or analyzed during the current study are available from the corresponding author on reasonable request.

\section{Authors' contributions}

AFS carried out the molecular and histological studies, performed the analysis and statistics of the molecular and histological data and interpretation of the data, and drafted the manuscript. PSK participated in the molecular studies and analysis. CAC participated in the design and conception of the study, participated in sample collection, helped in the analysis and interpretation of the data and helped draft the manuscript. SAD helped in the analysis and interpretation of the data and helped draft the manuscript. LAM participated in the conception of the study and coordinated the animal experiments. AHJ participated in the design and conception of the study, participated in sample collection, helped the interpretation of the data, and helped draft of the manuscript. SGK designed and conceived the study, participated in sample collection, helped the interpretation of the data, and helped draft of the manuscript. All authors read and approved the final manuscript.

\section{Competing interests}

The authors declare that they have no competing interests.

\section{Consent for publication}

Not applicable.

\section{Ethics approval and consent to participate}

The Institutional Animal Care and Use Committee at the University of California Davis approved all animal procedures, which were performed at the California National Primate Research Center (CNPRC), University of California, Davis.

\section{Author details}

'Division of Neonatology and Pulmonary Biology, Cincinnati Children's Hospital Medical Center, 3333 Burnet Ave, Cincinnati, OH 45229, USA ${ }^{2}$ Division of Immunobiology, Cincinnati Children's Hospital Medical Center, Cincinnati, OH, USA. ${ }^{3}$ Department of Anesthesia, Cincinnati Children's Hospital Medical Center, Cincinnati, OH, USA. ${ }^{4}$ California National Primate Research Center and Department of Pediatrics and Cell Biology and Human Anatomy, University of California, Davis, CA, USA.

\section{Received: 29 June 2016 Accepted: 25 August 2016}

\section{Published online: 06 September 2016}

\section{References}

1. lams JD, Romero R, Culhane JF, Goldenberg RL. Primary, secondary, and tertiary interventions to reduce the morbidity and mortality of preterm birth. Lancet. 2008;371:164-75.

2. Shatrov JG, Birch SC, Lam LT, Quinlivan JA, Mclntyre S, Mendz GL. Chorioamnionitis and cerebral palsy: a meta-analysis. Obstet Gynecol. 2010; 116:387-92.

3. Nasef N, Shabaan AE, Schurr P, et al. Effect of clinical and histological chorioamnionitis on the outcome of preterm infants. Am J Perinatol. 2013; 30(1):59-68.

4. Soraisham AS, Singhal N, McMillan DD, et al. A multicenter study on the clinical outcome of chorioamnionitis in preterm infants. Am J Obstet Gynecol. 2009;200(4):372. e1-6.

5. Soraisham AS, Trevenen C, Wood S, Singhal N, Sauve R. Histological chorioamnionitis and neurodevelopmental outcomes in preterm infants. J Perinatol. 2013;33(1):70-5.

6. Hagberg $\mathrm{H}$, Mallard C, Ferriero DM, Vanucci SJ, et al. The role of inflammation in perinatal brain injury. Nat Rev Neurol. 2015;11(4):192-208.

7. McRae A, Gilland E, Bona E, Hagberg H. Microglia activation after neonatal hypoxic-ischemia. Brain Res Dev Brain Res. 1995;84:245-52.

8. Gisslen T, Alvarez M, Wells $C$, et al. Fetal inflammation associated with minimal acute morbidity in moderate/late preterm infants. Arch Dis Child Fetal Neonatal Ed. 2016; in press.

9. Hoogland IC, Houbolt C, van Westerloo DJ, van Gool WA, van de Beek D. Systemic inflammation and microglial activation: systematic review of animal experiments. J Neuroinflammation. 2015;12:114.
10. Rousset $\mathrm{Cl}$, Chalon S, Bodard S, et al. Maternal exposure to LPS induces hypomyelination in the internal capsule and programmed cell death in the deep gray matter in newborn rats. Pediatr Res. 2006;59(3):428-33.

11. Cai Z, Lin S, Pang Y, Rhodes PG. Brain injury induced by intracerebral injection of interleukin-1 beta and tumor necrosis factor-alpha in the neonatal rat. Pediatr Res. 2004;56:377-84.

12. Pang $Y$, Tien $L T$, Zhu $H$, et al. Interleukin-1 receptor antagonist reduces neonatal lipopolysaccharide-induced long-lasting neurobehavioral deficits and dopaminergic neuronal injury in adult rats. In J Mol Sci. 2015;16(4): 8635-54.

13. Ellison VJ, Mocatta TJ, Winterbourn CC, Darlow BA, Volpe JJ, Inder TE. The relationship of CSF and plasma cytokine levels to cerebral white matter injury in the premature newborn. Pediatr Res. 2005:57:282-6.

14. Kunze M, Klar M, Morfeld CA, et al. Cytokines in noninvasively obtained amniotic fluid as predictors of fetal inflammatory response syndrome. Am J Obstet Gynecol. 2016; in press.

15. Strauss KL. Antiinflammatory and neuroprotective actions of COX2 inhibitors in the injured brain. Brain Behav Immun. 2008;22(3):285-98.

16. Kallapur SG, Presicce P, Rueda CM, Jobe AH, Chougnet CA. Fetal Immune response to chorioamnionitis. Semin Reprod Med. 2014;32(1):56-67.

17. Dulay AT, Buhimschi IA, Zhao G, et al. Compartimentalization of acute phase reactants interleukin-6, C-reactive protein and procalcitonin as biomarkers of intra-amniotic infections and chorioamnionitis. Cytokine. 2015;76(2):236-43.

18. Kallapur SG, Presicce P, Senthamaraikannan P, et al. Intra-amniotic IL-1 13 induces fetal inflammation in rhesus monkeys and alters the regulatory $T$ cell/IL-17 balance. J Immunol. 2013;191(3):1102-9.

19. Strackx E, Sparnaaji MA, Vlassaks E, Jellema R, Kuypers E, Vles JS, et al. Lipopolysaccharide-induced chorioamnionitis causes acute inflammatory changes in the ovine central nervous system. CNS Neurol Disord Drug Targets. 2015;14(1):77-84.

20. Kuypers E, Jellema RK, Ophelders DR, Dudink J, Nikiforou M, Wolfs TG, et al. Effects of intra-amniotic lipopolysaccharide and maternal betamethasone on brain inflammation in fetal sheep. PLoS One. 2013;8(12):e81644.

21. Semple BD, Blomgren K, Gimlin K, Ferriero DM, Noble-Haeusslein LJ. Brain development in rodents and humans: identifying benchmarks of maturation and vulnerability to injury across species. Prog Neurobiol. 2014:0:1-16.

22. Workman AD, Charvet CJ, Clancy B, Darlington RB, Finlay BL. Modeling transformations of neurodevelopmental sequences across mammalian species. J Neurosci. 2013;33:7368-83.

23. Institute of Medicine (US) Committee on Understanding Premature Birth and Assuring Healthy Outcomes; Behrman RE, Butler AS, editors. Preterm Birth: causes, consequences, and prevention. Washington (DC): National Academies Press (US); 2007. 12, Societal Costs of Preterm Birth. Available from: http://www.ncbi.nlm.nih.gov/books/NBK11358/

24. Goldenberg RL, Culhane JF, lams JD, Romero R. Epidemiology and causes of preterm birth. Lancet. 2008;371:75-84.

25. Pappas A, Kendrick DE, Shankaran $\mathrm{S}$, et al. Chorioamnionitis and early childhood outcomes among extremely low-gestational-age neonates. JAMA Pediatr. 2014:168(2):137-47.

26. Salas AA, Faye-Petersen OM, Sims B, et al. Histological characteristics of the fetal inflammatory response associated with neurodevelopmental impairment and death in extremely preterm infants. J Pediatr. 2013;163(3):652-7.

27. Poralla C, Hertfelder HJ, Oldenbur J, et al. Elevated interleukin-6 concentration and alterations of the coagulation system are associated with the development of intraventricular hemorrhage in extremely preterm. infants. Neonatology. 2012;102(4):270-5.

28. Lee Y, Kim HJ, Choi SJ, et al. Is there a stepwise increase in neonatal morbities according to histological stage(or grade) of acute chorioamnionitis and funisitis?: effect of gestational age. J Perinat Med. 2015:43(2):259-67.

29. Grether JK, Nelson KB. Maternal infection and cerebral palsy in infants of normal birthweight. JAMA. 1997;278(3):207-11.

30. Dammann O, Leviton A. Maternal intrauterine infection, cytokines, and brain damage in the preterm newborn. Pediatr Res. 1997;42(1):1-8.

31. Yoon BH, Romero R, Yang SH, et al. Interleukin-6 concentrations in umbilical cord plasma are elevated in neonates with white matter lesions with periventricular leukomalacia. Am J Obstet Gynecol. 1996;174(5):1433-40.

32. Hillier S, Witkin SS, Krohn MA, et al. The relationship of amniotic fluid cytokines and preterm delivery, amniotic fluid infection, histologic chorioamnionitis, and chorioamnion infection. Obstet Gynecol. 1993:81(6): 941-8. 
33. Banks WA, Kastin AJ, Broadwell RD. Passage of cytokines across the bloodbrain-barrier. Neuroimmunomodulation. 1995;2(4):241-8.

34. Gyoneva S, Ransohoff RM. Inflammatory reaction after traumatic brain injury: therapeutic potential of targeting cell-cell communication by chemokines. Trends Pharmacol Sci. 2015;36(7):471-80.

35. Savarin-Vuaillat C, Ransohoff RM. Chemokines and chemokine receptors in neurological disease: raise, retain, or reduce. Neurotherapeutics. 2007;4: 590-601.

36. Callahan MK, Williams KA, Ubogu EE, et al. Modulating CCR2 and CCL2 at the blood-brain barrier: relevance for multiple sclerosis pathogenesis. Brain. 2005:128:212-23.

37. Limperopoulos C, Soul JS, Gravreau L, et al. Late gestation cerebellar growth is rapid and impeded by premature growth. Pediatrics. 2005;115(3):688-95.

38. Shah DK, Anderon PJ, Carlin JB, et al. Reduction in cerebellar volumes in preterm infants: relationship to white matter injury and neurodevelopment at two years of age. Pediatr Res. 2006;60(1):97-102.

39. Hutton LC, Yan E, Yawno T, Castillo-Melendez M, Hirst JJ, Walker DW. Injury of the developing cerebellum: a brief review of the effects of endotoxin and asphyxial challenges in the late gestation sheep fetus. Cerebellum. 2014;13(6):777-86.

40. Volpe JJ. Cerebellum of the premature infant: rapidly developing, vulnerable, clinically important. J Child Neurol. 2009:24(9):1085-104.

41. Pierson CR, Folkerth RD, Trachtenberg FL, Drinkwater ME, Volpe JJ, Kinney HC. Gray matter damage is associated with periventricular leukomalacia. Acta Neuropathol. 2007;114:619-31.

42. Ligam P. Haynes RL, Folkerth RD, et al. Thalamic damage in periventricular leukomalacia: novel pathologic observations relevant to cognitive deficits in survivors of prematurity. Pediatr Res. 2009;65(5):524-9.

43. Fathali N, Ostrowski RP, Lekic T, et al. Cyclooxygenase-2 inhibition provides lasting protection against neonatal hypoxic-ischemic brain injury. Crit Care Med. 2010;38(2):572-8.

44. Harding DR, Humphries SE, Whitelaw A, Marlow N, Montgomery HE. Cognitive outcome and cyclo-oxygenase-2 gene $(-765 \mathrm{G} / \mathrm{C})$ variation in the preterm infant. Arch Dis Child Fetal Neonatal Ed. 2007;92(2):F108-12.

45. Fowlie PW, Davis PG. Prophylactic intravenous indomethacin for preventing mortality and morbidity in preterm infants. Cochrane Database Syst Rev. 2002:3:CD000174.

46. Bilimoria PM, Stevens B. Microglia function during brain development: new insights from animal models. Brain Res. 2015;1617:7-17.

47. Haynes RL, Folkerth RD, Keefe R, et al. Nitrosative and oxidative injury to premyelinating oligodendrocytes is accompanied by microglial activation in periventricular leukomalacia in the human premature infant. J Neuropathol Exp Neurol. 2003;62:441-50.

48. Vela JM, Molina-Holgado E, Arevalo-Martin A, Almazan G, Guaza C Interleukin-1 regulates proliferation and differentiation of oligodendrocyte progenitor cells. Mol Cell Neurosci. 2002;20:489-502.

49. Campbell LR, Pang Y, Ojeda NB, Zheng B, Rhodes PG, Alexander BT. Intracerebral lipopolysaccharide induces neuroinflammatory change and augmented brain injury in growth-restricted neonatal rats. Pediatr Res. 2012; 71(6):645-52.

50. Galvilanes AWD, Gantert M, Strackx E, et al. Increased EEG delta frequency corresponds to chorioamnionitis-related brain injury. Front Biosci (School Ed). 2010;2:432-8.

\section{Submit your next manuscript to BioMed Central and we will help you at every step:}

- We accept pre-submission inquiries

- Our selector tool helps you to find the most relevant journal

- We provide round the clock customer support

- Convenient online submission

- Thorough peer review

- Inclusion in PubMed and all major indexing services

- Maximum visibility for your research

Submit your manuscript at www.biomedcentral.com/submit

) Biomed Central 